\title{
Qualidade de vida de pacientes hemofílicos acompanhados em ambulatório de hematologia
}

\section{Quality of life of patients with hemophilia treated in a hematology clinic}

\author{
Altacílio A. Nunes ${ }^{1}$ \\ Blenda S. C. Rodrigues ${ }^{2}$ \\ Elisângela M. Soares ${ }^{3}$ \\ Sheila Soares ${ }^{4}$ \\ Sybelle S. C. Miranzi ${ }^{5}$
}

\begin{abstract}
O objetivo deste trabalho foi caracterizar a qualidade de vida de pacientes hemofilicos em acompanhamento ambulatorial em serviço especializado. Foi feita abordagem quantitativa da qualidade de vida $(Q V)$ de pacientes hemofilicos acompanhados em ambulatório de hematologia de um hemocentro regional. A coleta de dados foi realizada por meio de entrevistas utilizando-se o Whoqol-bref e questionário adicional com variáveis sociodemográficas e clínico-epidemiológicas. Para análise dos dados utilizaram-se o Epi-info 6.04d e o SPSS, cujos resultados foram expressos através de distribuição simples, medidas de tendência central e dispersão, proporções e correlação de Pearson entre facetas e domínios. Foram entrevistados 23 pacientes, com média de idade de 21 anos; todos moravam com familiares, 47,8\% eram residentes na cidade sede do hemocentro. Do total, 78,3\% eram solteiros, 69,6\% estudavam, sendo que $45,5 \%$ possuíam o $1^{\circ}$ grau incompleto e $82,6 \%$ não trabalhavam. A maioria $(91,3 \%)$ possuía hemofilia A. Quanto à avaliação da QV, 47,8\% responderam ser boa e $55 \%$ possuíam um bom nível de satisfação com a saúde. O domínio psicológico apresentou o maior escore médio e o menor foi o do domínio meio ambiente. Com esse estudo conseguiu-se salientar a magnitude de alguns problemas dos hemofilicos. Rev. Bras. Hematol. Hemoter.
\end{abstract}

Palavras-chave: Qualidade de vida; WHOQOL-bref; hemofilia.

\section{Introdução}

Hemofilia é uma coagulopatia hereditária recessiva ligada ao sexo, ocorrendo devida à falta ou produção defeituosa de moléculas dos fatores VIII ou IX da coagulação. A deficiência de fator VIII (Hemofilia A) é a mais frequente, ficando com $85 \%$ dos casos; já a deficiência do fator IX (Hemofilia B) corresponde a 15\% ${ }^{1,2} \mathrm{~A}$ incidência na população mundial é de 1/10.000 (pelo fator VIII) e de 1/30.000 (pelo fator IX) nascimentos do sexo masculino. É caracterizada pela ocorrência de hemorragias que aparecem espontaneamente ou em consequência de traumatismos leves e são comuns nas articulações. As hemorragias geralmente ocorrem nas grandes articulações, podendo causar muita dor, danos permanentes e incapacitantes se não forem tratados adequadamente. ${ }^{1-3}$ Segundo Ritterman, ${ }^{4}$ três componentes podem afetar os hemofílicos: o primeiro corresponde à atitude do indivíduo frente a sua enfermidade, seu sentimento de impotência frente aos sangramentos e a visão de não ser uma pessoa normal. O segundo refere-se ao contexto familiar em que, dentre as considerações, há a de que a hemofilia interfere na relação entre os pais e irmãos; o complexo de culpa que

\footnotetext{
${ }_{2}^{1}$ Médico. Professor do Departamento de Medicina Social - Faculdade de Medicina de Ribeirão Preto-USP - Ribeirão Preto-SP.

${ }_{3}^{2}$ Aluna do curso de Medicina da Universidade Federal do Triângulo Mineiro (UFTM) - Uberaba-MG.

${ }^{3}$ Enfermeira da Universidade Federal do Triângulo Mineiro (UFTM) - Uberaba-MG.

${ }_{5}^{4}$ Médica Hematologista. Professora Adjunta da Universidade Federal do Triângulo Mineiro (UFTM) - Uberaba-MG.

${ }^{5}$ Enfermeira. Professora Adjunta da Universidade Federal do Triângulo Mineiro (UFTM) - Uberaba-MG.

Faculdade de Medicina de Ribeirão Preto - Universidade de São Paulo.
}

Correspondência: Altacilio Nunes

Hospital das Clínicas - FMRP/USP - Departamento de Medicina Social

Av. Bandeirantes, 3900 - Monte Alegre

14049-900 - Ribeirão Preto-SP - Brasil

E-mail: altacilio@fmrp.usp.br

Doi: 
emerge das mães com superproteção do filho afetado e a frequente separação entre os pais. E, em terceiro lugar, encontra-se a sociedade que, possivelmente por sua falta de orientação sobre a doença, pode interferir de forma negativa na adaptação do paciente hemofílico em ambientes como escola, trabalho e lazer.

É relatado que a presença de doença crônica é inversamente proporcional à melhora da qualidade de vida $(\mathrm{QV})$ das pessoas ${ }^{5}$ e, segundo a Organização Mundial da Saúde (OMS), a QV é definida como: "a percepção do indivíduo de sua posição na vida no contexto da cultura e sistema de valores nos quais ele vive e em relação aos seus objetivos, expectativas, padrões e preocupações", ${ }^{2}$ apresentando significados distintos, para pessoas e situações diferentes. ${ }^{6,7}$ Os estudos que retratam a QV são indicadores que definem "a avaliação do impacto físico e psicossocial que as enfermidades, disfunções ou incapacidades podem acarretar para as pessoas acometidas, permitindo um melhor conhecimento do paciente e de sua adaptação à condição". ${ }^{8}$

Motivados em contribuir com a melhoria do cuidado prestado a esses pacientes conjeturou-se que um estudo da QV possibilitaria mudanças necessárias para a obtenção de bem-estar desse grupo, descrever a magnitude dos problemas da população estudada, prever condições para sua reabilitação e abordar o paciente de maneira integral, enfocando simultaneamente sua sintomatologia clínica com seu aspecto psicossocial. Os objetivos do presente estudo foram avaliar a QV de uma população de pacientes com hemofilia em acompanhamento em um serviço ambulatorial de um hemocentro regional e descrever o perfil sociodemográfico e clínico-epidemiológico destes.

\section{Casuística e Método}

Trata-se de um estudo transversal, realizado com pacientes hemofílicos em acompanhamento no Ambulatório de um Hemocentro Regional (HR) durante o ano de 2006.

Critérios de elegibilidade, inclusão e exclusão

Dos 36 pacientes cadastrados, fizeram parte da pesquisa 23 pacientes em acompanhamento no HR, que atenderam os critérios de inclusão. Os demais sujeitos não foram incluídos na pesquisa por possuírem endereço desatualizado e não estarem em acompanhamento regular. Foram critérios de inclusão não apresentar comprometimento cognitivo severo, e como critério de exclusão apresentar idade inferior a 8 anos e não concordar em participar da pesquisa.

Para a coleta de dados, empregou-se um instrumento que constou de duas partes: a primeira destinada à caracterização dos dados sociodemográficos, clínico-epidemiológicos (tipo de hemofilia, prática de atividade física, uso de fator liofilizado, comorbidades) e a segunda parte contendo o questionário WHOQOL-bref ${ }^{7}$ autoaplicado.

\section{Análise estatística}

Para armazenamento e análise dos dados empregou-se o programa SPSS, sendo este utilizado para aplicar a sintaxe WHOQOL-bref, na avaliação dos escores do instrumento de QV e posteriores correlações a partir do coeficiente de Pearson. A mensuração da QV é proporcional ao escore, quanto maior o valor do escore, melhor a QV. O nível de significância para todas as análises foi de $5 \%(\mathrm{p}<0,05)$.

\section{Aspectos éticos}

O Projeto de Pesquisa foi apresentado ao Comitê de Ética em Pesquisa da Universidade Federal do Triângulo Mineiro e os dados só foram coletados após aprovação do mesmo. Todos os pacientes participantes e/ou seus responsáveis leram e assinaram um Termo de Consentimento Livre e Esclarecido.

\section{Resultados}

A média de idade dos pacientes foi de 21 anos, variando entre 9 e 50 anos. O grupo mais frequente quanto ao estado civil foi o de solteiros, com 78,3\%. Dos sujeitos que compuseram a pesquisa, $69,6 \%$ estudavam, sendo que o nível educacional predominante foi o $1^{\circ}$ grau incompleto com $45,5 \%$ dos casos.

Dos entrevistados, $82,6 \%$ responderam que não trabalhavam, $13 \%$ possuíam emprego formal e legalizado. Sessenta e nove por cento dos entrevistados referiram não realizar atividade física.

Ao se perguntar sobre a utilização de fator liofilizado nos últimos trinta dias, $43,5 \%$ relataram não ter utilizado. Dos que fizeram o uso, $82,6 \%$ referiram não considerar alto seu consumo. Quanto às comorbidades, $60,9 \%$ referiram não apresentar nenhuma, quatro possuíam hemoartrose e três artrite reumatoide. Quanto ao tipo de hemofilia, 91,3\% possuem hemofilia A e 8,7\% hemofilia B.

Tabela 1. Distribuição descritiva das questões 1 e 2 e da QVG, em pacientes hemofílicos, 2006

\begin{tabular}{lllcc}
\hline \multicolumn{1}{c}{ Questão } & \multicolumn{1}{c}{$\begin{array}{c}\text { Opções de } \\
\text { resposta }\end{array}$} & N & Média & $\begin{array}{c}\text { Escore } \\
\text { médio }\end{array}$ \\
\hline Q1 & 1. Muito ruim & 1 & & \\
"Como você avalia & $\begin{array}{l}\text { 2. Ruim } \\
\text { sua QV?" }\end{array}$ & 1 & & \\
& $\begin{array}{l}\text { 3. Nem ruim } \\
\text { nem boa }\end{array}$ & 3 & 4 & 18,5 \\
& $\begin{array}{l}\text { 4. Boa } \\
\text { 5. Muito boa }\end{array}$ & 11 & & \\
Q2 & 1. Muito satisfeito & 1 & & \\
"Quão satisfeito & 2. Insatisfeito & 3 & & \\
você está com & 3. Nem satisfeito & 6 & 4 & 15,8 \\
sua saúde?" & $\quad$ nem insatisfeito & & & \\
& 4. Satisfeito & 9 & & \\
QVG & 5. Muito satisfeito & 4 & & \\
\hline
\end{tabular}


Tabela 2. Distribuição dos escores médios, correlação de cada questão de pacientes hemofílicos, 2006

\begin{tabular}{lcccc}
\hline \multicolumn{1}{c}{ Questão } & $\begin{array}{c}\text { Escore } \\
\text { médio }\end{array}$ & Correlação & $\begin{array}{c}\text { Escore médio } \\
\text { do domínio }\end{array}$ & $p$ \\
\hline Domínio Físico & & & 64,9 & \\
\hline Q3 (dor e desconforto) & 59,2 & 0,60 & & $<0,001$ \\
Q4 (dependência de medicações e & 62,6 & 0,41 & & \\
$\quad$ tratamentos) & 75,6 & $0,80^{* *}$ & & \\
Q10 (energia e fadiga) & 78,2 & $0,73^{*}$ & & \\
Q15 (mobilidade) & 79,2 & $0,79^{*}$ & & \\
Q16 (sono e repouso) & 80,0 & $0,92^{* *}$ & & \\
Q17 (atividade cotidiana) & 68,6 & $0,62^{*}$ & & \\
Q18 (capacidade de trabalho) & & & 73,6 & \\
\hline Domínio Psicológico & 78,2 & $0,74^{*}$ & & \\
\hline Q5 (sentimentos positivos) & 82,6 & $0,86^{\star *}$ & & \\
Q6 (espiritualidade) & 69,6 & $0,78^{*}$ & & \\
Q7 (pensar, aprender, memória e & & & & \\
$\quad$ concentração) & 84,4 & $0,78^{*}$ & & \\
Q11 (imagem corporal e aparência) & 77,4 & $0,73^{*}$ & \\
Q19 (autoestima) & 80,8 & 0,33 & \\
Q26 (sentimentos negativos) & & & \\
\hline
\end{tabular}

\begin{tabular}{|c|c|c|c|}
\hline \multicolumn{2}{|l|}{ Domínio Relações Sociais } & \multicolumn{2}{|c|}{70,6} \\
\hline Q20 (relações pessoais) & 87,0 & $0,81^{* *}$ & $<0,001$ \\
\hline Q21 (atividade sexual) & 67,0 & $0,85^{* *}$ & \\
\hline Q22 (apoio social) & 75,6 & $0,84^{\star \star}$ & \\
\hline \multicolumn{2}{|l|}{ Domínio Meio Ambiente } & \multicolumn{2}{|c|}{59,5} \\
\hline Q8 (segurança física e proteção) & 66,0 & $0,70^{*}$ & $<0,001$ \\
\hline $\begin{array}{l}\text { Q9 (ambiente físico, poluição, ruído, } \\
\text { transito e clima) }\end{array}$ & 65,2 & $0,75^{\star}$ & \\
\hline Q12 (recursos financeiros) & 54,8 & $0,65^{*}$ & \\
\hline $\begin{array}{l}\text { Q13 (oportunidade de adquirir } \\
\text { novas habilidades) }\end{array}$ & 67,0 & $0,82^{* *}$ & \\
\hline $\begin{array}{l}\text { Q14 (oportunidades de } \\
\text { recreação/lazer) }\end{array}$ & 62,6 & $0,80^{\star *}$ & \\
\hline Q23 (ambiente no lar) & 80,0 & $0,80^{* *}$ & \\
\hline Q24 (cuidados de saúde e sociais) & 72,2 & 0,60 & \\
\hline Q25 (transporte) & 72,2 & $0,80^{* *}$ & \\
\hline
\end{tabular}

*correlação moderada ${ }^{* *}$ correlação forte

\section{Avaliação da QV}

QV geral

A QV geral (QVG) refere-se às médias entre as duas primeiras perguntas do WHOQOL-bref; em Q1 avalia-se a QV de modo geral e em Q2 avalia-se a satisfação com própria saúde.

Com relação à Q1, 47,8\% responderam ser boa e 30,7\%, muito boa. Com relação à Q2, a satisfação com a própria saúde, $39,1 \%$ dos hemofílicos demonstraram-se satisfeitos e $17,4 \%$ muito satisfeitos. Ou seja, 55\% dos hemofílicos possuíam um bom nível de satisfação com a saúde. Utilizando-se a escala de 4 a 20 , a QV geral correspondeu a 15 pontos.

\section{Domínios}

Na Tabela 2 estão representados os escores médios, na escala de 0 a 100 , e os desvios-padrão obtidos nos domínios. O domínio psicológico apresentou o maior escore médio.

\section{Domínio fisico}

Observa-se que as questões do domínio físico apresentaram escore médio acima de 59 na escala de 0 a 100, sendo a faceta com maior escore médio e correlação a de atividade da vida cotidiana. De acordo com os dados, as facetas do domínio físico apresentaram correlação positiva e estatisticamente significativa com o escore médio, sendo que as facetas que apresentaram correlação forte foram a de atividade da vida cotidiana $(r=0,92 ; p<0,000)$, seguida de energia e fadiga $(r=0,08 ; p<0,000)$, ou seja, estas foram as que mais contribuíram para a média do domínio. Em relação às facetas que compuseram esse domínio, a "dor e o desconforto", para $47,8 \%$ dos entrevistados, dificultam bastante a realização de suas atividades; entretanto, $34,8 \%$ referiram haver muito pouca interferência, sendo essa a faceta com menor escore médio.

Quanto à "dependência de tratamento ou de medicação", a qual se apresentou como o segundo menor escore e menor correlação com o escore médio do domínio, $34,8 \%$ dos sujeitos responderam necessitar mais ou menos do serviço de saúde.

Ao se perguntar sobre "energia para realizar as atividades diárias", 39,1\% responderam apresentar energia completamente. Quanto à "capacidade de locomoção", $56,5 \%$ referiram ser boa e $21,7 \%$ muito boa. Em relação à "satisfação com o sono", 43,5\% referiram estar muito satisfeitos e $34,8 \%$ satisfeitos, ou seja, neste estudo, mais de $78 \%$ dos sujeitos estão satisfeitos com o sono. No que diz respeito à "satisfação com a capacidade de desempenhar as atividades do dia-a-dia", 43,5\% referiram estar muito satisfeitos. Na avaliação de sua "capacidade de trabalho", 30,4\% demonstraram-se satisfeitos e muito satisfeitos com esse quesito.

\section{Domínio psicológico}

Observa-se que as questões do domínio psicológico apresentaram escore médio acima de 69 pontos se carac- 
terizando como o domínio com melhor resultado. As facetas que apresentaram maior escore médio, ou seja, boa interferência na QV foram a de imagem corporal seguida pela de espiritualidade. Pode-se verificar que todas as facetas do domínio psicológico apresentaram correlação positiva e estatisticamente significativa com o escore médio do domínio. A questão que obteve maior correlação foi Q6 (espiritualidade, religião e crenças pessoais) $(r=0,86 ; \mathrm{p}<0,000)$, embora o maior escore médio tenha sido Q11 (imagem corporal e aparência). Observou-se que $60,9 \%$ dos sujeitos da pesquisa referiram aproveitar bastante à vida, o que contribui para a elevação da QV. Quanto à "espiritualidade, religião e crenças pessoais", $47,8 \%$ referiram fazer sentido de forma extrema.

No que diz respeito à "concentração", 54,5\% referiram se concentrar bastante, sendo que essa faceta apresentou o menor escore médio. Quanto à "imagem corporal", apresentaram-se completamente satisfeitos $52,2 \%$ dos sujeitos e muito satisfeitos $26,1 \%$. Quanto à "satisfação consigo mesmo", 43,5\% referiram estar satisfeito. No que diz respeito à frequência com que tinham "sentimentos negativos", 47,8\% referiram apresentar tais sentimentos algumas vezes.

\section{Relações sociais}

O domínio relações sociais apresentou o segundo maior escore médio entre os outros. As três facetas do domínio apresentaram alta correlação positiva e estatisticamente significativa com o escore médio, havendo concordância nos valores das questões. Sendo a faceta "relações pessoais" a que apresentou maior média conforme observado, nela incluem amigos, parentes, conhecidos e colegas. Dos entrevistados, $52,2 \%$ a consideraram como sendo muito satisfatória. Com relação à "satisfação com a vida sexual", 36,4\% responderam, respectivamente, estarem satisfeitos e muito satisfeitos.

\section{Meio ambiente}

O domínio meio ambiente teve escores médios acima de 54, sendo esse o que apresentou menor escore médio entre todos os domínios; a média encontrada foi menor em 5,8 pontos. Em relação à correlação entre as questões e o escore médio, verificou-se que as oito facetas apresentam correlações positivas e significativas; a faceta que apresentou maior correlação foi (Q13) oportunidade de adquirir novas informações $(r=0,82 ; p<0,000)$.

A "segurança física" foi considerada intermediária para $39,1 \%$ dos sujeitos e como bastante seguro para $30,4 \%$. Quanto aos "recursos financeiros", 26,1\% referiram possuir muito pouco, e $21,7 \%$ nada e média quantidade.

No que se refere à "oportunidade de adquirir novas informações", 34,8\% referiram apresentar média e muita oportunidade, respectivamente. Com relação às "oportunidades de participação em recreação e lazer", 26,1\% referiram apresentar poucas oportunidades, e $21,7 \%$ apresentaram muita e completamente.
O "acesso aos serviços de saúde" obteve a menor correlação com a média do domínio meio ambiente, sendo que, para $34,8 \%$ dos sujeitos, foi considerado como intermediário, e, para 30,4\%, muito satisfatório. O "transporte", para $43,5 \%$ é muito satisfatório, e para $21,7 \%$, satisfatório, o que sugere estar interferindo positivamente em vários aspectos da QV como, por exemplo, acesso aos serviços de saúde e ao lazer.

\section{Discussão}

Através deste estudo foi possível caracterizar atributos comuns aos pacientes hemofílicos e ainda conhecer aspectos não médicos inerentes a eles. $\mathrm{Na}$ análise dos dados sociodemográficos observou-se que a maioria dos pacientes, procedia de cidades próximas, o que demonstra uma situação peculiar para o portador de hemofilia, que apresenta uma dependência em termos de distância geográfica e de disponibilidade de um centro especializado de tratamento. ${ }^{2}$ Quanto ao estado civil, notou-se que a maioria $(78,30 \%)$ era composta de indivíduos solteiros, diferentemente de dados de outro estudo, em que $83 \%$ eram casados ou amasiados, ${ }^{2,9}$ enquanto, em estudo realizado pelo Hospital das Clínicas da Universidade de São Paulo, constatou-se que 69\% dos pacientes eram solteiros. ${ }^{9}$ Essas diferenças podem ser justificadas pelas variações das faixas etárias, sendo a média de idade dos pacientes tratados neste hemocentro de 21 anos, em Campinas de 39 anos $^{2}$ e na USP com $81,2 \%$ na faixa entre 16 e 30 anos. ${ }^{9}$

Dos indivíduos que compuseram a pesquisa, $69,6 \%$ estudavam, sendo que o nível educacional predominante foi o $1^{\circ}$ grau incompleto, com $45,5 \%$. Estudos internacionais demonstram que uma característica psicossocial dos hemofílicos é o absenteísmo escolar, ${ }^{7}$ sendo observado, em trabalho realizado pela Universidade de Campinas, que 50\% dos pacientes relataram interferência da doença na vida escolar. ${ }^{2}$ Em estudo da Faculdade de Medicina da Universidade de São Paulo, $57,4 \%$ dos pacientes possuíam $1^{\circ}$ e $2^{\circ}$ graus incompletos; 9 já no HR, onde esta pesquisa foi realizada, a porcentagem foi de $72,8 \%$, o que pode ser justificado por $74 \%$ dos selecionados serem compostos por pessoas menores de 24 anos de idade. A falta de escolaridade favorece as desigualdades sociais e, por conseguinte, o baixo nível de QV. Uma pesquisa realizada em Madrid demonstra que $70 \%$ dos hemofílicos obtiveram quociente intelectual similar ao restante da população, sendo o atraso escolar justificado pelo absenteísmo escolar. ${ }^{10}$ Ainda observou-se que $82,6 \%$ dos sujeitos não trabalhavam. Pode-se supor que estes dados possam estar correlacionados à baixa escolaridade e idade dos entrevistados, o que difere da realidade observada com os pacientes de Campinas, em que $73 \%$ possuíam emprego regular, já que no estudo realizado pela USP, $51 \%$ dos sujeitos estavam inativos temporária ou definitivamente. ${ }^{2,9}$ 
Quanto à atividade física, 69,6\% responderam não estar participando de nenhuma no momento, o que remete ao fato de que, no passado, pelos riscos temidos, os hemofílicos eram desestimulados à prática de atividade física. ${ }^{11} \mathrm{Com}$ a introdução do fator liofilizado de uso domiciliar para tratamento profilático, tornou-se possível que hemofílicos pudessem ter uma vida com participação ativa em casa, escola, trabalho, esportes e lazer, ${ }^{12}$ sendo a atividade física considerada como benéfica a eles. ${ }^{13}$ Em estudo realizado nos Países Baixos demonstrou-se que não houve diferença na participação em atividades físicas entre hemofílicos, que faziam a profilaxia com o fator liofilizado e controles saudáveis. ${ }^{14}$

Quanto à qualidade de vida geral dos pacientes, $47,8 \%$ julgaram-na como "boa" e 30,7\%, como "muito boa". Considerando estudos realizados utilizando o mesmo questionário para outras doenças, percebeu-se que em pacientes com Diabetes tipo II, 54,4\% consideraram sua QV como nem ruim nem boa. ${ }^{6}$ Em outra pesquisa, $74,5 \%$ dos pacientes hemofílicos manifestaram uma atitude positiva frente à sua enfermidade. ${ }^{10}$

Nos quatro domínios que compõem o questionário, notou-se que o psicológico foi o de maior pontuação, indicando ser esse o fator que mais influencia na QV de hemofílicos, seguido, respectivamente, pelos domínios relações sociais e físico. Na avaliação do domínio físico, houve uma contradição de pensamentos referentes à avaliação do impacto da dor, uma vez que, para $47,8 \%$ dos entrevistados, dificultava bastante a realização de suas atividades; entretanto, 34,8\% referiram haver muito pouca interferência, sendo essa a faceta com menor escore médio. Deve-se lembrar, porém, que a dor é particularizada pelo indivíduo conforme as suas experiências prévias com esse sintoma, sendo essa avaliação subjetiva e individual. ${ }^{15} \mathrm{~A}$ dor é experiência cotidiana nas instituições de saúde e suas vivências resultam em alterações biológicas, psicossociais e sofrimento. A mesma leva a prejuízos nos relacionamentos interpessoais, no trabalho, no sono e na movimentação. A dor, quando não tratada adequadamente, afeta a $\mathrm{QV}$ dos doentes e de seus cuidadores em todas as dimensões: física, psicológica, social e espiritual, ${ }^{16} \mathrm{e}$, por se tratar de experiência tão particular, supõe-se que esta possa ser a causa da divergência entre os pacientes.

Quanto à "dependência de tratamento ou de medicação", em que foram obtidas respostas nos cinco níveis possíveis, desde "nada" até "extremamente", essa distribuição das frequências das respostas pode ser vinculada a diferentes graus de acometimento da hemofilia. Em pesquisa realizada por Caio et al., $65 \%$ dos sujeitos consideraram um problema importante a dependência de um centro de tratamento. ${ }^{2}$

No quesito "locomoção", 78,2\% referiram ser "boa" ou "muito boa", o que interfere de forma favorável na QV do indivíduo por englobar outras atividades da vida cotidiana, como acesso aos serviços de saúde, lazer, frequência escolar, entre outras. Ainda deve-se lembrar que estes dados estão compatíveis com as comorbidades, onde quatro hemofílicos referiram possuir hemoartrose e três, artrite reumatoide.

Ritterman et al. demonstraram que $20 \%$ a $40 \%$ da população mundial apresentam problemas relacionados ao sono, sendo preocupante, pois tais problemas interferem de forma negativa na QV dos indivíduos. ${ }^{5}$ Porém, neste estudo, mais de $78 \%$ dos sujeitos estão satisfeitos com o sono.

$\mathrm{Na}$ avaliação de sua "capacidade de trabalho", 30,4\% demonstraram-se satisfeitos e muito satisfeitos com esse quesito. A capacidade de trabalho recebe influências de diversos fatores tais como aspectos sociodemográficos, estilo de vida, processo de envelhecimento, exigências do trabalho, sendo a saúde considerada como um dos principais fatores. ${ }^{17}$ Os hemofílicos demonstram sua capacidade de competir no mercado de trabalho, observa-se a necessidade de adequação de suas funções e liberação para o acompanhamento ambulatorial interprofissional para a manutenção de suas potencialidades.

Já em relação às facetas que constituem o domínio psicológico, observou-se que foram as que obtiveram os maiores escores, ou seja, uma interferência positiva na qualidade de vida dos pacientes hemofílicos. Alguns estudos apontam para uma associação benéfica entre religiosidade e saúde mental, dessa forma atuando de maneira positiva na QV. ${ }^{17}$ Ainda sobre esse domínio, a grande maioria responde estar "muito satisfeita" ou "completamente satisfeita" em relação à sua imagem. Segundo a literatura, os hemofílicos possuem um reforço negativo em sua aceitação da imagem corporal devido à angústia demonstrada pelos pais e parentes. ${ }^{9}$ Mas os dados encontrados neste hemocentro demonstraram uma não interferência na aceitação da imagem corporal, que pode ser justificada pela menor pressão social sofrida pelos homens, levando a uma possível melhor aceitação da sua imagem ou mesmo por uma participação familiar diferenciada. ${ }^{18}$

No que diz respeito à frequência com que tinham "sentimentos negativos", 47,8\% referiram apresentar tais sentimentos algumas vezes e $37,8 \%$ referiram nunca ter apresentado, sendo esse dado importante, pois o otimismo está relacionado com as expectativas que as pessoas possuem acerca de seu futuro. Existe um consenso de que as pessoas otimistas enfrentam de forma melhor seus problemas, possuem menos estresse e dessa forma adoecem menos. ${ }^{19,20}$

Sobre o domínio relações sociais, o qual apresentou o segundo melhor escore, obteve-se que a maioria $(52,2 \%)$ dos pacientes o considerou no mais alto padrão possível, ou seja, "muito satisfatório". No entanto, a família, estrutura muitas vezes sólida e acolhedora, parece ser abalada com o nascimento de uma criança com algum grau de deficiência. $\mathrm{Na}$ hemofilia, estudos demonstram não ser diferente, afetando as mães que, muitas vezes, sentem-se culpadas pelo fato do filho apresentar a doença. Ao se avaliar a QV de crianças e 
jovens com hemofilia, os pais avaliaram a QV de seus filhos de forma ligeiramente mais baixa do que as crianças. ${ }^{20}$

Quanto à "satisfação com a vida sexual", $36,4 \%$ responderam respectivamente estarem satisfeitos e muito satisfeitos, o que se mostra compatível com a faixa etária deste estudo. Na pesquisa conduzida na Unicamp com pacientes hemofílicos, $13 \%$ referiram interferência da doença na atividade sexual. ${ }^{2}$

No domínio meio ambiente, o de menor pontuação entre os demais, subentende-se que haja influência do meio em que se vive na QV dos entrevistados. O meio ambiente em que habitamos se constitui em um espaço de construção e desenvolvimento da saúde da família, ${ }^{21}$ sendo que esse deverá apresentar, além de equipamentos urbanos básicos, redes de apoio que possibilitem hábitos psicossociais saudáveis. (Cohen SC et al. 2003. Disponível em: http:// www.abrasco.org.br/gts/gt/promocao/habitacaosaudável/ psf.pdf) ${ }^{22}$ Outra questão abordada em relação ao meio ambiente foi a segurança física, considerado bastante seguro para 30,4\%. É relevante observar que a segurança física relatada como intermediária pode ser decorrente da violência e da criminalidade que afetam a população, independente da classe social, raça, religião e sexo. Sendo, nos dias de hoje, as taxas de criminalidade consideradas graves, o que pode ser observado com esse tipo de estudo de QV dos indivíduos, independentemente de se possuir ou não alguma doença. ${ }^{22}$

Por fim, em relação ao quesito acesso aos serviços de saúde, $34,8 \%$ dos hemofílicos o consideraram como intermediário e 30,4\% muito satisfatório; o ideal, no caso da hemofilia, seria ter acesso muito satisfatório em grandes proporções, tendo em vista as particularidades da doença, que, muitas vezes, requerem assistência imediata, portanto, não podendo haver qualquer fator que dificulte o livre acesso à assistência.

\section{Conclusões}

De modo geral, os hemofílicos apresentam boa QV, sendo prejudicada por baixa renda, dificuldade de acesso aos serviços de saúde, dor e oportunidades de trabalho. O presente estudo evidencia a importância do tema, pois a hemofilia é doença que traz consequências biopsicossociais, o que remete à necessidade de incorporar os indivíduos portadores da doença no ambiente social, proporcionando incentivo ao estudo e esportes, acesso aos serviços de saúde, assim como a inserção dos mesmos no mercado de trabalho. Delinear programas específicos de tratamento com abordagem multiprofissional, o fornecimento domiciliar de fator liofilizado, assim como desenvolvimento de ações preventivas de quadros hemorrágicos, vislumbrando a melhoria da QV.

\section{Abstract}

The objective of this work was to characterize the quality of life of hemophilic patients being followed up in a specialized service. A cross-sectional study of hemophilic patients in a Regional Blood Bank of Brazil was carried out to evaluate their quality of life. The data were obtained by interviews employing the WHO QOL-brief questionnaire, which was analyzed using SPSS and Epi-info 6.04d computer programs. Twenty-three male patients with a mean age of 21 years old were interviewed. All reported that they live with their families, $47.8 \%$ were residents in Uberaba, $78.3 \%$ were single, $69.6 \%$ were students with $45.5 \%$ having a low level of education and $82.6 \%$ did not work. Of the 23 cases, $91.3 \%$ had hemophilia A. In respect to quality of life, $47.8 \%$ responded that their quality of life was good and 55\% had a good level of satisfaction with their health. The psychological dominion presented the highest average score and the environment presented the lowest. This study highlights the magnitude of some problems of hemophilic patients. Rev. Bras. Hematol. Hemoter.

Key words: Quality of life; WHOQOL-bref; haemophilia.

\section{Referências Bibliográficas}

1. Brasil. Ministério da Saúde. Manual de tratamento das coagulopatias hereditárias. Brasília DF, 2005

2. Caio MV, Silva RBP, Magna LA, Ramalho AS. Genética comunitária e hemofilia em uma população brasileira. Cad Saúde Pública 2001; 17:535-605

3. Pacheco RLR, Alencar PGC, Yoshiyasu GA, Veiga MT. Cirurgia Ortopédica em Pacientes Hemofílicos. Rev Bras Ortop 2002; 37: 108-13.

4. Weinmann AF, Schoof JM, Thompson AR. Clinical correlates among 49 families with hemophilia A and factor VIII gene inversions. Am J Hematol. 1996;51(3):192-9.

5. Ritterman MK. Hemophilia in context: adjunctive hypnosis for families with a hemophiliac member. Fam Process. 1982;21 (4):469-76.

6. Aguilar PIDSG. Qualidade de vida em pessoas com diabetes mellitus tipo 2 [Dissertação de Doutorado]. Ribeirão Preto: Escola de Enfermagem de Ribeirão Preto, Universidade de São Paulo; 2004.

7. Fleck MPA, Louzada S, Xavier M, Chachamovich E, Vieira G, Santos L, et al. Aplicação da versão em português do instrumento abreviado de avaliação da qualidade de vida "WHOQOL-bref". Rev Saúde Pública 2000; 34:178-83.

8. Minayo MCS, Hartz ZMA, Buss PM. Qualidade de vida e saúde: um debate necessário. Ciênc Saúde Coletiva 2000; 5:7-18.

9. Michelone APC, Santos VLCG. Qualidade de vida de adultos com câncer colorretal com e sem ostomia. Rev Latin Am Enferm 2004; 12: $875-83$.

10. Vizcaíno CC, Vizcaíno G, Carrizo E, Vizcaíno MA, Sarmiento S, Carruyo JV. Actitud de los indivíduos adultos com hemofilia hacia su enfermedad. Invest Clin 2004; 45: 257-67.

11. Broderick CR, Herbert RD, Latimer J, Curtin JA, Selvadurai HC. The effect of an exercise intervention on aerobic fitness, strength and quality of life in children with haemophilia (ACTRN012605000224628). BMC Blood Disord. 2006;6:2.

12. Fiala KA, Riternour DM. Medial care for athletes with haemophilia. Athl Ther Today 2004;9:16-9. 
13. World Federation of Hemolhilia. Guidance on Physical Activity ans Sports for People with Haemophilia and Related Disorders. Montreal, 1998.

14. Heijnen L, Mauser-Bunschoten EP, Roosendaal G. Participation in sports by Dutch persons with haemophilia. Haemophilia. 2000; 6(5):537-46.

15. Pimenta CAM, Cruz DALM. Instrumentos para avaliação da dor: o que há de novo em nosso meio. Arq Bras Neurocir 1998; 17(1):15-24

16. Ferrell BR, Wisdom C, Rhiner M, Alletto J. Pain management as a quality of care outcome. J Nurs Qual Assur. 1991;5(2):50-8.

17. Panzini RG, Rocha NS, Bandeira DR, Fleck MPA. Qualidade de vida e espiritualidade. Rev Psiq Clín 2007; 34: 105-115.

18. Graham MA, Eich C, Kephart B, Peterson D. Relationship among body image, sex, and popularity of high school students. Percept Mot Skills. 2000;90(3 Pt 2):1187-93.

19. Fiumi, A. Orientação familiar: o profissional fisioterapeuta segundo percepção das mães de crianças portadoras de paralisia cerebral [Dissertação de Mestrado]. São Paulo: Universidade Presbiteriana Mackenzie; 2003.

20. Pollak E, Mühlan H, VON Mackensen S, Bullinger M; HaemoQoL Group The Haemo-QoL Index: developing a short measure for health-related quality of life assessment in children and adolescents with haemophilia. Haemophilia. 2006;12(4):384-92.

21. Cohen SM, Cynamon SE, Kligerman DC, Assumpção RF. Habitação Saudável no Programa Saúde da Família (PSF): uma estratégia para as políticas públicas de saúde e ambiente. Rev Ciên Saúde Coletiva 2004;9:807-813

22. Cohen SC et al. 2003. Habitação saudável no SUS. Uma estratégia de ação para o PSF: uma incorporação do conceito de habitação saudável na política pública de saúde. ENSP, Rio de Janeiro; 2003. P. 807-13.

Avaliação: Editor e dois revisores externos

Conflito de interesse: sem conflito de interesse

Recebido: 01/02/2009

Aceito: 08/04/2009 